\title{
Reconfiguring Smart Structures Using Phase Space Connections
}

\author{
C R McInnes, T J Waters \\ Department of Mechanical Engineering, University of Strathclyde, Glasgow, G1 1XJ, \\ $\mathrm{UK}$ \\ E-mail: colin.mcinnes@strath.ac.uk
}

\begin{abstract}
Structures are traditionally designed to be stable. However, unstable configurations (such as buckling) can in principle be controlled in smart structures using embedded sensors and actuators. In this paper we explore a new means of reconfiguring smart structures by connecting multiple unstable configurations. Methods from dynamical systems theory are used firstly to identify sets of unstable configurations in a simple smart structure model, and then to connect them through so-called heteroclinic connections in the phase space of the problem. The instability inherent in the structure is then actively utilised to provide an effective new way of transitioning between configurations of the structure in a controlled manner.
\end{abstract}

\section{Introduction}

Smart structures offer the possibility of active control of mechanical systems through the use of embedded sensors and actuators. Applications range from active vibration control of small amplitude displacements through to active shape control at large deformations (e.g. $[1,2,3])$. Other more ambitious applications include improving the buckling limit of beams under compressive load. For active control of buckling, continuous sensing and actuation is required to ensure the integrity of the structure past the classical, critical buckling limit. In such applications computational effort replaces mechanical strength. The embedded controller must then cope with potentially violent instability, as opposed to small amplitude displacements for vibration control (e.g. $[4,5])$.

The active control of unstable smart structures has been investigated by Hogg and Huberman using an agent-based approach to suppress instability [6]. These ideas were extended by Guenther et al. to consider the possible benefits of transitions between unstable states, relative to transitions between stable states [7, 8]. Using relatively simple, but qualitatively interesting models, these investigations provide new insights into the dynamics of unstable smart structures. In particular, the concept of exploiting instability is raised as a means of reducing the energy requirements for transitions between configurations of the structure. Numerical experiments demonstrated a reduction in both the energy and time required to transition between unstable states relative to transitions between stable states. 
In this paper, the exploitation of instability is linked to the powerful concept of phase space connections from modern dynamical systems theory [9]. Firstly, a set of equilibrium configurations will be identified in a simple model of a reconfigurable smart structure. A reconfigurable smart structure is defined here as a mechanical system which has the ability to change its kinematic configuration between a finite set of equilibria (stable or unstable). It will be assumed that the simple reconfigurable structure used later possesses embedded sensors and actuators which will allow the structure to be actively controlled. In particular, it will be assumed that the structure can be stabilised at naturally unstable equilibria through the use of active control. It will then be shown that a subset of unstable, equal energy configurations can be connected, allowing highly efficient reconfiguration of the entire structure. The development of this central idea will exploit aspects of modern dynamical system theory, which again will be illustrated using a simple, representative model of an unstable smart structure. It is our intention in this paper to introduce and explore a new concept, rather than provide a detailed analysis using a high fidelity model of a real structure.

In general, non-linear dynamical systems typically posses a number of equilibria which may be connected through paths in the phase space of the system. In particular, equilibria with both stable and unstable manifolds may be connected through so-called heteroclinic connections [9]. Here, a manifold is defined as a surface embedded in phase space. The unstable manifold then represents the family of trajectories diverging from an equilibrium point, while the stable manifold represents the family of trajectories asymptotically approaching an equilibrium point. A heteroclinic connection is formed if the unstable manifold of an equilibrium point intersects the stable manifold of another distant equilibrium point in phase space. These phase space structures have important applications in diverse fields such as mechanics [10], astrodynamics [11] and fluids [12]. More complex phase space structures, termed heteroclinic networks, can then be formed from an assembly heteroclinic connections [13].

Heteroclinic connections will investigated here as a rigorous means of enabling transitions between unstable configurations of a reconfigurable smart structure. For a real mechanical system, heteroclinic connections will correspond to phase space trajectories whose projection into configuration space connects the unstable configurations (equilibria) of the structure. As will be seen, unstable equilibria can be found which lie on the same energy surface in phase space. Therefore, if heteroclinic connections between unstable, equal energy equilibria can be identified, trajectories exist between these configurations which in principle do not require the addition of or dissipation of energy. The intersection of invariant manifolds in phase space can then be used as a novel means of connecting different, equal energy unstable configurations, leading to highly efficient reconfiguration of the structure. We note here that compliant structures with multiple equilibria have been investigated for some time, but that unstable equilibria are largely ignored, with only transitions between stable equilibria deemed important (e.g. [14, 15]).

As will be seen, the use of heteroclinic connections between unstable, equal energy 
equilibria can in principle be extremely efficient compared to reconfiguring a structure between stable configurations. A transition between stable configurations requires the input of, and then dissipation of energy to cross the potential barrier separating the stable equilibria. The work done to cross the barrier requires the input of energy, while the subsequent dissipation of that energy to reach the new stable equilibrium generates waste heat. In comparison, the use of heteroclinic connections between unstable, equal energy equilibria only requires that the effect of dissipation during the reconfiguration process is compensated for. This dissipation is a path integral along the heteroclinic connection through phase space, and can be overcome using a suitable controller. Clearly, the energy required to actively control the instability must also be sufficiently small for the concept to be of benefit. However, for frequently actuated devices the accumulated work done in reconfiguring between stable equilibria will be significant, while the duration the system spends in active control at unstable equilibria will be small. Further work will investigate this issue in detail.

Potential applications of the phase space connection concept include use in MEMStype devices which require frequent switching of compliant components to reduce mean power consumption and waste heat dissipation. For example, previous work has demonstrated the existence of multiple equilibria in MEMS cantilevered beams [16] and torsional devices [17] with electrostatic forces, while MEMS-scale bistable beam mechanisms have been fabricated [18]. On a more speculative level, switched nano-mechanical devices have been considered for reversible computing which require frequent, rapid mechanical transitions [19]. Other applications include power reduction in smart structures used in rapidly actuated control surfaces for active flow control and the efficient reconfiguration of large deployable spacecraft antennae [20, 21]. In general, the use of heteroclinic connections between unstable equilibria is likely to be of benefit for power and energy constrained applications, such as aerospace, and for compliant structures requiring frequent reconfiguration, such as MEMS devices.

\section{Smart structure model}

\subsection{Single mass problem}

In order to investigate the use of heteroclinic connections to reconfigure unstable smart structures, a simple representative model of a naturally unstable structure will be defined. Firstly, a simple model of a beam under compressive load will be considered. The essential behaviour of the beam can be captured by representing the inertia of the beam as a single lumped mass and the stiffness of the beam by two linear springs. We therefore consider a beam clamped at both ends, as shown in figure 1, represented as a mass $m$ connected to two linear springs of stiffness $k$ and natural length $l$. If the displacement of the mass is defined by $x$, (such that $x \leq \sqrt{l^{2}-d^{2}}$ ), while the springs are separated by $2 d$, such that $d<l$, it can be shown that dynamics of the problem are described by 


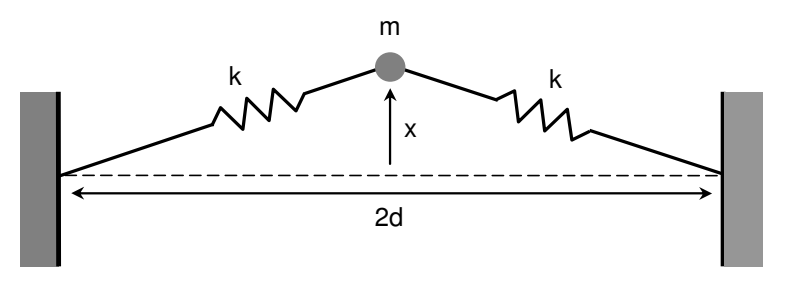

Figure 1. 1 degree-of-freedom buckling beam model comprising a single lumped mass $m$ with spring constant $k$ and displacement $x$ from (unstable) equilibrium. The springs are pinned at both ends and the displacement is assumed to be constrained to the vertical.

$$
\begin{aligned}
& \dot{x}=v \\
& m \dot{v}=-2 k x\left(1-\frac{l}{\sqrt{x^{2}+d^{2}}}\right)
\end{aligned}
$$

The non-linear term in equation (2) can be expanded by assuming $x / d \ll 1$ to obtain

$$
\begin{aligned}
& \dot{x}=v \\
& m \dot{v}=2 k\left(\frac{l}{d}-1\right) x-\frac{k l}{d^{3}} x^{3}+\ldots
\end{aligned}
$$

Then, a non-dimensional position coordinate $q=\sqrt{l / d^{3}} x$ and non-dimensional time $\tau=t / \sqrt{m / k}$ can be defined. In this simple model, the position coordinate $q$ represents the displacement of the beam from its undeformed state with corresponding momentum coordinate $p$ such that $(q, p) \in \mathbf{R}^{2}$. We note that although equation (4) has been derived assuming $x / d \ll 1$, we will place no restriction on $q$ in the subsequent analysis. The simple cubic nonlinearity in equation (4) will suffice to provide the required qualitative behaviour in the model, without undue algebraic complexity. The qualitative non-linear model for the beam is therefore defined by

$$
\begin{aligned}
& \dot{q}=p \\
& \dot{p}=\mu q-q^{3}
\end{aligned}
$$

The free parameter $\mu=2(l / d-1)$ is used as a measure of the compressive load acting on the beam. Clearly, if the structure is in tension $(l<d)$ then $\mu<0$ while if the structure in in compression $(l>d)$ then $\mu>0$ with the critical buckling load corresponding to $\mu=0$. It can be seen that for $\mu<0$, equation (6) admits a single real equilibrium solution $(\dot{p}=0)$ at $\widetilde{q}_{0}=0$ corresponding to an undeflected beam in tension. For $\mu>0$, equation (6) admits 3 equilibria defined as $\widetilde{q}_{0}=0, \widetilde{q}_{1}=+\sqrt{\mu}$ and $\widetilde{q}_{2}=-\sqrt{\mu}$, corresponding to a symmetric buckled configuration. A supercriticial bifurcation then occurs when $\mu$ changes sign. 
The change to the qualitative behaviour of the system detailed above can be seen through the use of an effective potential for the problem $V(q, \mu)$ such that $\dot{p}=-\partial V(q, \mu) / \partial q$. The potential can then be defined as

$$
V(q, \mu)=-\frac{1}{2} \mu q^{2}+\frac{1}{4} q^{4}
$$

The change to the number and type of turning points of $V(q, \mu)$ can be seen in figure 2 , again with the supercriticial bifurcation occurring at $\mu=0$. For $\mu<0$ there is a single global minimum at $\widetilde{q}_{0}$ defined by $\partial V(q, \mu) / \partial q=0$ with $\partial^{2} V(q, \mu) / \partial q^{2}>0$ while for $\mu>0$ there are 3 turning points with a local maximum at $\widetilde{q}_{0}$ with $\partial^{2} V(q, \mu) / \partial q^{2}<0$ and local minima at $\widetilde{q}_{1}$ and $\widetilde{q}_{2}$ with $\partial^{2} V(q, \mu) / \partial q^{2}>0$. It is clear that $\widetilde{q}_{0}$ becomes unstable when the two new (stable) equilibrium points $\widetilde{q}_{1}$ and $\widetilde{q}_{2}$ appear.

The stability properties of the equilibria can also be determined from the eigenvalues $\lambda$ which are found from linearisation of equation (6). The eigenvalue spectrum is found to be $\pm \sqrt{\mu}, \pm i \sqrt{2 \mu}$ and $\pm i \sqrt{2 \mu}$ for $\widetilde{q}_{0}, \widetilde{q}_{1}$, and $\widetilde{q}_{2}$ respectively. Again, it can be seen that for $\mu<0, \widetilde{q}_{0}$ is linearly stable $(\operatorname{Re}(\lambda)=0)$ while for $\mu>0, \widetilde{q}_{0}$ becomes unstable $(\operatorname{Re}(\lambda)>0)$ while the two new equilibria $\widetilde{q}_{1}$ and $\widetilde{q}_{2}$ are linearly stable. The onset of buckling at $\mu=0$ is again identified as the transition from $\widetilde{q}_{0}$ to one of the two new stable equilibria at $\widetilde{q}_{1}$ or $\widetilde{q}_{2}$. The supercriticial bifurcation at $\mu=0$ can then be associated with buckling at some critical compressive load. The full bifurcation diagram for this single mass problem is shown in figure 3.

Lastly, we note that while equation (6) has similarities to a pitchfork bifurcation, the classical pitchfork bifurcation is in fact defined for first order systems [22]. However, with strong dissipation (over-damped system) equation (6) can be transformed to first order. Adding linear dissipation parameterised by $\beta$, equation (6) becomes

$$
\begin{aligned}
& \dot{q}=p \\
& \dot{p}=\mu q-q^{3}-\beta p
\end{aligned}
$$

Then, if $\beta \gg 1$, equation (9) reduces to the first order equation $\dot{q}=(1 / \beta)\left(\mu q-q^{3}\right)$, which has the same equilibria as equation (6) and represents a classical pitchfork bifurcation. Physically, this represents a rapid transition to the buckled state after the bifurcation. With modest dissipation added to the second order system in equation (9), the stable equilibria $\widetilde{q}_{1}$ and $\widetilde{q}_{2}$ are no longer centres with purely imaginary eigenvalues, but spirals with complex eigenvalues with negative real part. The effect of dissipation on the reconfiguration problem presented here will be discussed later.

\subsection{Chain of coupled masses}

We now consider a coupled problem with a chain of $N$ masses, using the same functional form of non-linearity as defined in equation (7) above. This coupling of neighbouring elements will then be used to define a simple model of an unstable smart structure. As with the models of Hogg et al., we capture the qualitative behaviour of a naturally 


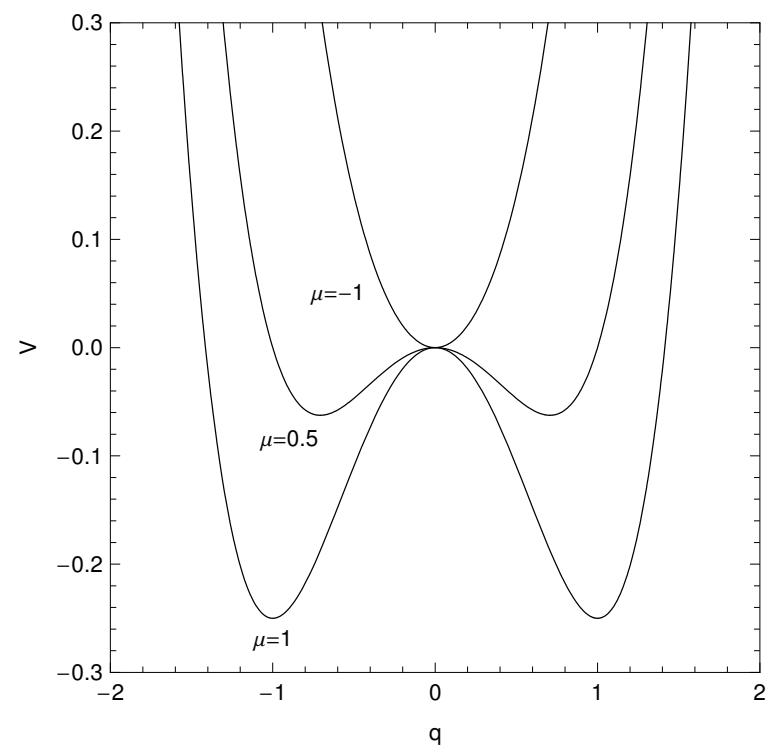

Figure 2. Effective potential $V(q, \mu)$ for a 1 mass chain with a single lumped mass. Single equilibrium $\widetilde{q}_{0}$ for $\mu<0$ with two new equilibria $\widetilde{q}_{1}$ and $\widetilde{q}_{2}$ appearing after the supercriticial bifurcation at $\mu=0$.

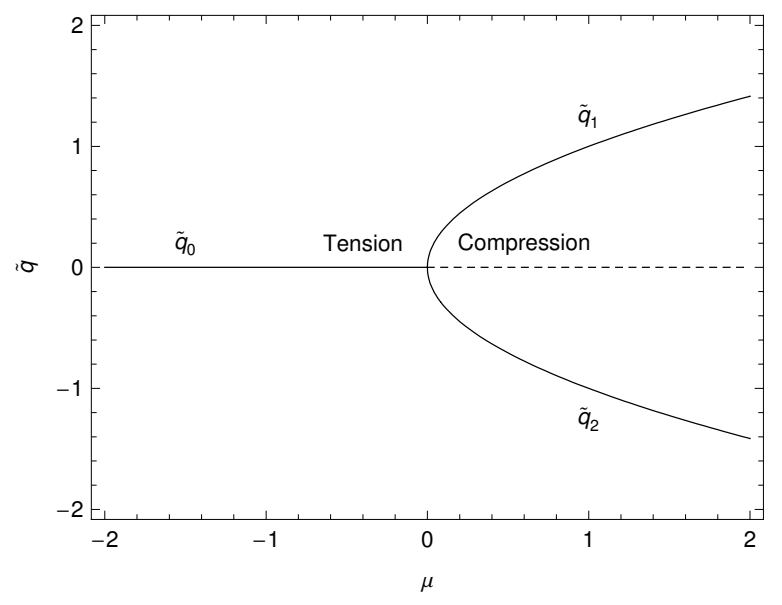

Figure 3. Bifurcation diagram for a 1 mass chain for $-2 \leq \mu \leq 2$, solid line: stable equilibria $(\operatorname{Re}(\lambda)=0)$, dashed line: unstable equilibria $(\operatorname{Re}(\lambda)>0)$. The equilibrium at $\widetilde{q}_{0}=0$ becomes unstable for $\mu>0$, creating stable equilibria $\widetilde{q}_{1}$ and $\widetilde{q}_{2}$.

unstable structure $[6,7,8]$. It will be assumed at first that the dynamics of the problem are conservative with no dissipation. The effect of dissipation will be discussed later.

From equation (7), the generation of the potential can be generalised to an arbitrary pair of neighbouring masses $i-1$ and $i$ as

$$
V\left(q_{i-1}, q_{i}, \mu_{i}\right)=-\frac{1}{2} \mu_{i}\left(q_{i-1}-q_{i}\right)^{2}+\frac{1}{4}\left(q_{i-1}-q_{i}\right)^{4}
$$

This potential provides a de-stabilising linear force at small displacements and a stabilising, non-linear restoring force at large displacements. It will be assumed later 
that the displacements $q_{1,2}$ can be sensed and that the coupling parameters $\mu_{1-3}$ can be manipulated to effect active control over the structure. Manipulating the coupling coefficients is equivalent to manipulating the natural length of the springs in the model. In principle, a spring fabricated from shape memory alloy could posses this property. It can be seen that equation (10) has similarities with the classical problem of oscillations in a chain of coupled masses [23], but with instability since $\mu_{i}>0$. However, the interaction of the de-stabilising quadratic term with the stabilising quartic term will yield families of both stable and unstable equilibria.

The global configuration of the unstable structure is now defined by the set $\mathbf{q}=\left\{q_{i}\right\}$ $(i=1-N)$ with an associated set of momenta $\mathbf{p}=\left\{p_{i}\right\}(i=1-N)$ such that $(\mathbf{q}, \mathbf{p}) \in \mathbf{R}^{2 N}$. The behaviour of the chain of masses can be described by a Hamiltonian $H(\mathbf{q}, \mathbf{p}, \mu)=T(\mathbf{p})+V(\mathbf{q}, \mu)$ for kinetic energy $T(\mathbf{p})$ and potential $V(\mathbf{q}, \mu)$ where

$$
\begin{aligned}
& T(\mathbf{p})=\frac{1}{2}\left\|\mathbf{p}^{2}\right\| \\
& V(\mathbf{q}, \mu)=\sum_{i=1}^{N+1}-\frac{1}{2} \mu_{i}\left(q_{i-1}-q_{i}\right)^{2}+\frac{1}{4}\left(q_{i-1}-q_{i}\right)^{4}
\end{aligned}
$$

with the Dirichlet boundary conditions $q_{0}=0$ and $q_{N}=0$, so that the chain is pinned at both ends. The dynamics of the problem (phase flow) are now defined by Hamilton's equations of the form

$$
\begin{aligned}
& \dot{\mathbf{q}}=\nabla_{p} H(\mathbf{q}, \mathbf{p}, \mu) \\
& \dot{\mathbf{p}}=-\nabla_{q} H(\mathbf{q}, \mathbf{p}, \mu)
\end{aligned}
$$

where $\nabla_{q}=\partial / \partial \mathbf{q}$ and $\nabla_{p}=\partial / \partial \mathbf{p}$. These can be written in compact form using $\mathbf{z}=(\mathbf{q}, \mathbf{p}) \in \mathbf{R}^{2 N}$ as

$$
\dot{\mathbf{z}}=J \cdot \nabla_{z} H(\mathbf{z}, \mu)
$$

where the symplectic matrix $J$ is defined by

$$
J=\left(\begin{array}{cc}
0 & I \\
-I & 0
\end{array}\right)
$$

In general, a set of equilibrium configurations $E$ for equation (15) can be found defined as $\widetilde{\mathbf{z}}_{i}(i=1-N)$. However, since the potential is independent of $\mathbf{p}$, the set $E$ is obtained from $\nabla_{q} V(\mathbf{q}, \mu)=0$. This condition results in an algebraic equation, the solution of which which yields the set of equilibria $E$ defined as $\widetilde{\mathbf{q}}_{i}(i=1-N)$. In general $E$ will contain both linearly stable equilibria $E^{s}(\operatorname{Re}(\lambda)=0)$ and unstable equilibria $E^{u}$ $(\operatorname{Re}(\lambda)>0)$ for some eigenvalue spectrum $\lambda$.

In general, the linear stability of the set $E$ can be determined through linearisation of Hamilton's equations in the neighbourhood of each equilibrium point in $E$. Defining a displacement $\xi$ from equilibrium $\widetilde{\mathbf{z}}$ such that $\xi=\mathbf{z}-\widetilde{\mathbf{z}}$, a Taylor expansion of equation (15) provides

$$
\dot{\xi}=J \cdot \nabla_{z}^{2} H(\widetilde{\mathbf{z}}, \mu) \xi
$$




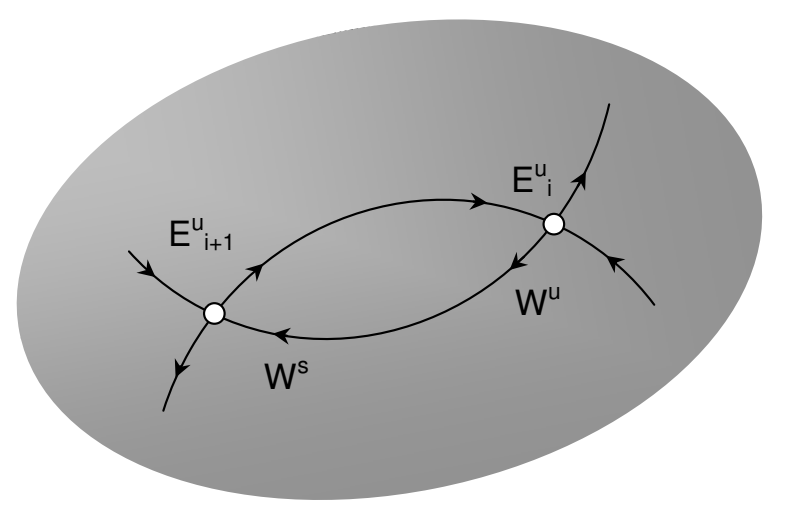

Figure 4. Heteroclininc connection between two distant equilibria $E_{i}^{u}$ and $E_{i+1}^{u}$ on the same energy surface in phase space, formed by the intersection of the unstable manifold $W^{u}$ of $E_{i}^{u}$ and stable manifold $W^{s}$ of $E_{i+1}^{u}$

Then, the eigenvalue spectrum $\lambda$ of $E$ is found from the resulting characteristic polynomial obtained from

$$
\left\|J \cdot \nabla_{z}^{2} H(\widetilde{\mathbf{z}}, \mu)-\lambda \cdot I\right\|=0
$$

For the Hamiltonian problem considered here, a set of linearly stable equilibria $E^{s}$ are expected with conjugate imaginary eigenvalues along with a set of unstable equilibria $E^{u}$ with real eigenvalues of opposite sign [9]. These so-called hyperbolic points represent trajectories in phase space which recede from an equilibrium point along an unstable manifold $W^{u}(\operatorname{Re}(\lambda)>0)$ or approach an equilibrium point along a stable manifold $W^{s}$ $(\operatorname{Re}(\lambda)<0)$. If the unstable manifold $W^{u}$ of an equilibrium point $E_{i}^{u}$ intersects the stable manifold $W^{s}$ of a distant equilibrium point $E_{i+1}^{u}$ in phase space, then a heteroclinic connection exists between the equilibria. In general, if $\psi(t)$ is a phase space trajectory there exists a heteroclinic connection between $\widetilde{\mathbf{z}}_{i}$ and $\widetilde{\mathbf{z}}_{i+1}$ if $\psi(t) \rightarrow \widetilde{\mathbf{z}}_{i}$ as $t \rightarrow-\infty$ and $\psi(t) \rightarrow \widetilde{\mathbf{z}}_{i+1}$ as $t \rightarrow+\infty$. Here, we are particularly interested in the set of hyperbolic points $E^{u}$ which exist on the same energy surface $W$ in phase space, as shown in figure

4. Heteroclinic connections between these points do not in principle require the addition of or dissipation of energy, with significant practical benefits for reconfiguring the smart structure.

\section{Heteroclinic connections}

\subsection{Two mass chain}

In order to explore the possibility of reconfiguring a smart structure using heteroclinic connections, a two mass chain with three linear springs will now be considered with the springs clamped at both ends, as shown in figure 5. It will be assumed that the masses are constrained to move along the vertical direction and, again, that the dynamics are conservative. The Hamiltonian of this two mass problem can then be constructed from the kinetic and potential energy as 


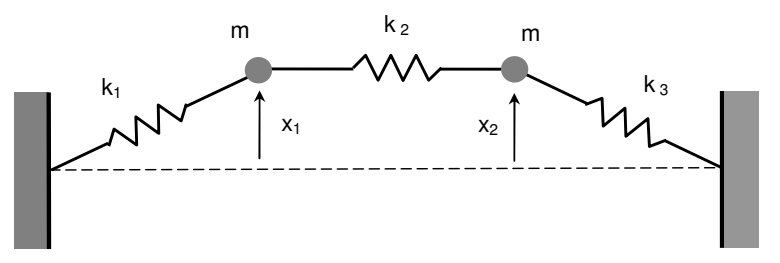

Figure 5. 2 degree-of-freedom buckling beam model comprising two lumped masses $m$ with spring constants $k_{1,2}$ and displacements $x_{1,2}$ from (unstable) equilibrium. The springs are pinned at both ends and the displacement is assumed to be constrained to the vertical.

$$
\begin{aligned}
T(\mathbf{p})= & \frac{1}{2}\left(p_{1}^{2}+p_{2}^{2}\right) \\
V(\mathbf{q}, \mu)= & -\frac{1}{2} \mu_{1} q_{1}^{2}-\frac{1}{2} \mu_{2}\left(q_{1}-q_{2}\right)^{2}-\frac{1}{2} \mu_{3} q_{2}^{2}+\frac{1}{4} q_{1}^{4} \\
& +\frac{1}{4}\left(q_{1}-q_{2}\right)^{4}+\frac{1}{4} q_{2}^{4}
\end{aligned}
$$

The dynamics of the chain are then obtained from Hamilton's equations, where now $(\mathbf{q}, \mathbf{p}) \in \mathbf{R}^{4}$. However, since the kinetic energy is clearly independent of $\mathbf{q}$, it can be seen from equation (14) that the dynamics are described by $\dot{\mathbf{p}}=-\nabla_{q} V(\mathbf{q}, \mu)$ so that

$$
\begin{aligned}
& \dot{q_{1}}=p_{1} \\
& \dot{p_{1}}=\mu_{1} q_{1}-q_{1}^{3}+\mu_{2}\left(q_{1}-q_{2}\right)-\left(q_{1}-q_{2}\right)^{3} \\
& \dot{q_{2}}=p_{2} \\
& \dot{p_{2}}=\mu_{3} q_{2}-q_{2}^{3}-\mu_{2}\left(q_{1}-q_{2}\right)+\left(q_{1}-q_{2}\right)^{3}
\end{aligned}
$$

Calculating the gradient of the potential such that $\nabla_{q} V(\mathbf{q}, \mu)=0$, yields two simultaneous algebraic equations whose solution defines the set of equilibria $E$. However, from equations (22) and (24) it can be seen immediately that

$$
\dot{p_{1}}+\dot{p_{2}}=q_{1}\left(\mu_{1}-q_{1}^{2}\right)+q_{2}\left(\mu_{3}-q_{2}^{2}\right)
$$

so that we expect equilibria at $(0,0),\left(\sqrt{\mu_{1}}, \sqrt{\mu_{3}}\right)$ and $\left(-\sqrt{\mu_{1}},-\sqrt{\mu_{3}}\right)$. However, when the coupling constants are equal, such that $\mu_{1-3}=\mu$, the equilibria merge to form a continuous ring defined by $\mu\left(q_{1}+q_{2}\right)-\left(q_{1}^{3}+q_{2}^{3}\right)=0$. This rotational symmetry is then broken for $\mu_{2}>\mu_{1}$ to form 5 equilibria, as will be discussed later. Also, since in general $\dot{p_{1}}+\dot{p_{2}} \neq 0$, the total momentum of the system is not conserved. While the total energy is conserved, the absence of another integral of motion for such a 2-degree-of-freedom system suggests that the system is not integrable.

Solving $\nabla_{q} V(\mathbf{q}, \mu)=0$ yields five equilibria for $\mu_{1-3}>0$. The location of the equilibria are listed in table 1 for $\mu_{1}=1, \mu_{2}=1.5$ and $\mu_{3}=1$ along with the eigenvalue spectrum associated with each equilibria, again obtained by linearisation. It can be seen 
that the system possesses 1 unstable equilibrium $E_{0}$, where the potential has a global maximum, 2 unstable equilibria $E_{1}$ and $E_{2}$ where the potential has a saddle and 2 stable equilibria $E_{3}$ and $E_{4}$ where the potential has a global minimum, as can be seen in figure 6. The corresponding shape of the structure associated with each of these 5 equilibrium configurations shown in figure 7 . It can be seen from table 1 that $E_{0}$ has the highest potential $V$, corresponding to the two masses being undeflected, with both springs in compression. $E_{1}$ and $E_{2}$ then have equal potential which is higher than $E_{3}$ and $E_{4}$. For the unstable equilibria $E_{1}$ and $E_{2}$, only the central spring is in compression and can in principle relax to the lower energy equilibria at $E_{3}$ and $E_{4}$ where both springs are extended. We note that since the Hamiltonian $H=T(\mathbf{p})+V(\mathbf{q}, \mu)$ is constant, the volume of phase space in $\mathbf{R}^{4}$, and its projection to configuration space in $\mathbf{R}^{2}$, is constrained by the requirement that $T(\mathbf{p}) \geq 0$.

It can be shown that the stability properties of the equilibria are a function of the coupling parameters $\mu_{1-3}$. In particular, the stability properties of $E_{1,2}$ and $E_{3,4}$ swap for $\mu_{2}>\mu_{1}$, as will be seen later. It can also be shown that the location of $E_{1}$ and $E_{2}$ are independent of $\mu_{2}$ while the location of $E_{3}$ and $E_{4}$ is a function of $\mu_{2}$. Therefore, fixing $\mu_{1}$ and $\mu_{3}$, a bifurcation diagram can be constructed. Although only $\mu_{1-3}>0$ is considered in the subsequent analysis, for completeness the bifurcation diagram is shown for $-2 \leq \mu_{2} \leq 2$ in figure 8 . It can be seen that the stability properties of $E_{1,2}$ and $E_{3,4}$ swap for $\mu_{2}>\mu_{1}$, as noted above.

Since the unstable equilibria $E_{1}$ and $E_{2}$ lie on the same energy surface, if a heteroclinic connection can be found between $E_{1}$ and $E_{2}$ the structure can in principle be reconfigured from $E_{1}$ to $E_{2}$ without working being done, again in the absence of dissipation. The change in energy for the reconfiguration $\delta V=V\left(\widetilde{\mathbf{q}}_{1}\right)-V\left(\widetilde{\mathbf{q}}_{2}\right) \approx 0$. If the structure in figure 6 is at the stable equilibrium $E_{3}$, it can transition to the other stable equilibrium at $E_{4}$ only by crossing the potential barrier at $E_{1}$. Therefore, the change in energy for reconfiguration to $E_{4}$ is $\delta V=V\left(\widetilde{\mathbf{q}}_{3}\right)-V\left(\widetilde{\mathbf{q}}_{1}\right) \approx-0.39$, assuming that the energy input to cross the potential barrier at $E_{1}$ is dissipated to finally reach $E_{4}$. If frequent reconfigurations of the structure are required, it is clear that the use of heteroclinic connections between unstable equilibria may be significantly more efficient. We note however, that work is in principle required required to stabilise the structure when operating at the unstable configuration at $E_{1}$. However, by exploiting the change in stability properties of $E_{1}$ and $E_{2}$ close to $\mu_{2}=\mu_{1}$ it will be possible to provide passive stability to $E_{1}$ and $E_{2}$, as will be discussed later.

In order to explore possible connections between these unstable equilibria, their stable and unstable manifolds must be investigated. Firstly the eigenvectors associated with each eigenvalue are found. For the 2 unstable equilibria $E_{1}$ and $E_{2}$ there exists pair of real eigenvalues of opposite sign. Associated with these eigenvalues are eigenvectors $\mathbf{u}^{\mathbf{s}}$ and $\mathbf{u}^{\mathbf{u}}$ corresponding to the eigenvalues $\lambda=-1$ and $\lambda=+1$ respectively. These eigenvectors are tangent to the stable manifold $W_{s}$ and the unstable manifold $W_{u}$ attached to the equilibria. Therefore, the stable and unstable manifolds can be mapped by integrating forwards/backwards from a neighbourhood of the equilibrium point in 


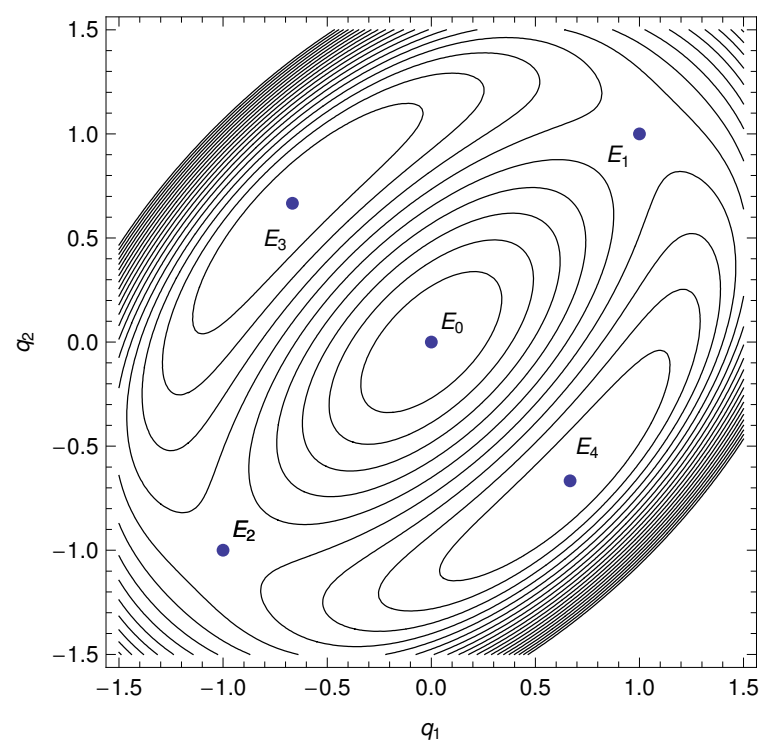

Figure 6. Effective potential $V(\mathbf{q}, \mu)$ for a two mass chain with $\mu_{1}=1, \mu_{2}=1.5$ and $\mu_{3}=1$ yielding 5 equilibria ( 3 unstable equilibria $E_{0}, E_{1}$ and $E_{2}$, and 2 stable equilibria $E_{3}$ and $E_{4}$ ).
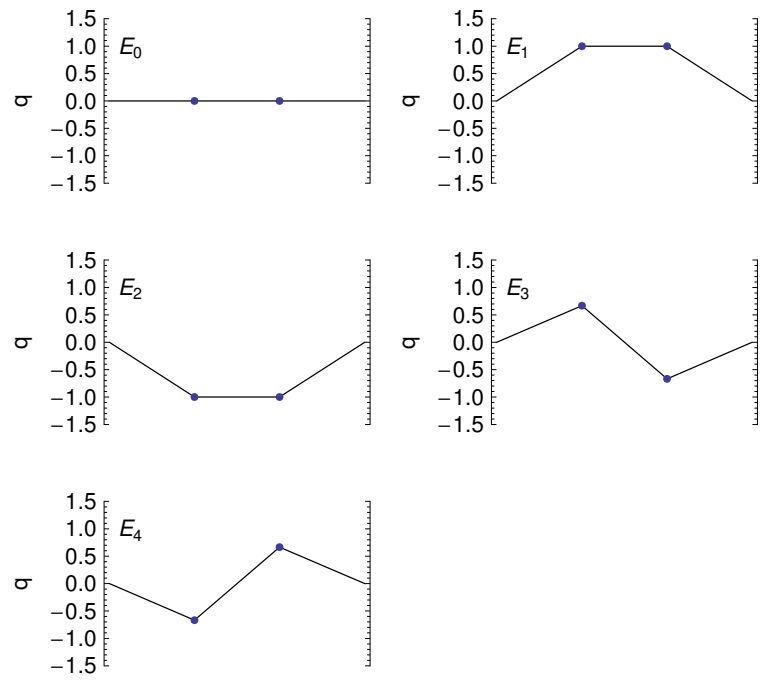

Figure 7. Equilibria for a two mass chain with unstable equilibria $E_{0}, E_{1}$ and $E_{2}$ and stable equilibria $E_{3}$ and $E_{4}$. The unstable symmetric pair $E_{1}$ and $E_{2}$ have equal potential $V$.

Table 1. Stability properties of the 5 equilibria of a two mass chain with $\mu_{1}=1$, $\mu_{2}=1.5$ and $\mu_{3}=1$

\begin{tabular}{lllllll}
\hline Point & $\widetilde{q}_{1}$ & $\widetilde{q}_{2}$ & $\lambda_{1,2}$ & $\lambda_{3,4}$ & $V$ & Type \\
\hline$E_{0}$ & 0 & 0 & \pm 1 & \pm 2 & 0 & Saddle $\times$ Saddle \\
$E_{1}$ & 1 & 1 & $\pm \sqrt{2} i$ & \pm 1 & $-1 / 2$ & Saddle $\times$ Centre \\
$E_{2}$ & -1 & -1 & $\pm \sqrt{2} i$ & \pm 1 & $-1 / 2$ & Saddle $\times$ Centre \\
$E_{3}$ & $-2 / 3$ & $2 / 3$ & $\pm 1 / \sqrt{3} i$ & $\pm 2 \sqrt{2} i$ & $-8 / 9$ & Centre $\times$ Centre \\
$E_{4}$ & $2 / 3$ & $-2 / 3$ & $\pm 1 / \sqrt{3} i$ & $\pm 2 \sqrt{2} i$ & $-8 / 9$ & Centre $\times$ Centre \\
\hline
\end{tabular}




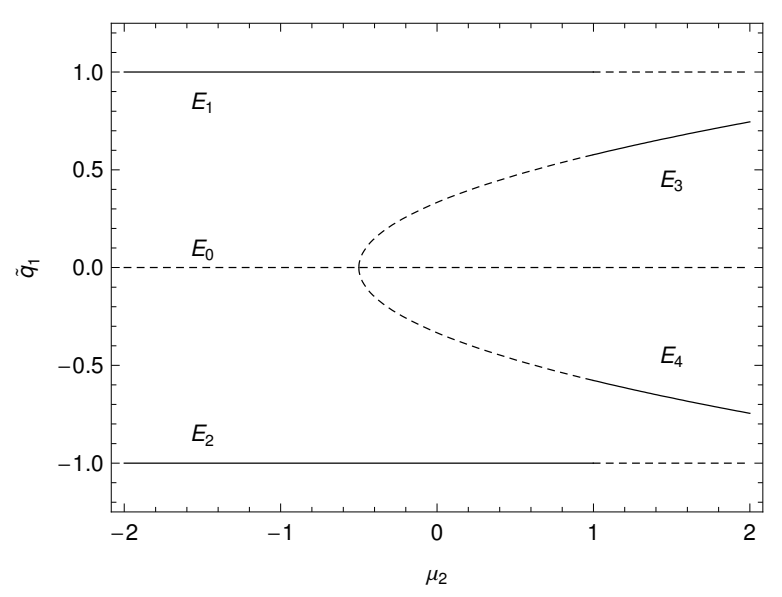

Figure 8. Bifurcation diagram for a two mass chain. Projection of the location of the equilibria onto the $q_{1}$ axis for $\mu_{1}=1, \mu_{3}=1$ and $-2 \leq \mu_{2} \leq 2$, solid line: stable equilibria $(\operatorname{Re}(\lambda)=0)$, dashed line: unstable equilibria $(\operatorname{Re}(\lambda)>0)$.

phase space with initial conditions

$$
\begin{aligned}
& \mathbf{z}^{s}=\mathbf{z}^{e} \pm \epsilon \mathbf{u}^{s} \\
& \mathbf{z}^{u}=\mathbf{z}^{e} \pm \epsilon \mathbf{u}^{u}
\end{aligned}
$$

for $\epsilon \ll 1$. If the manifolds intersect then a heteroclinic connection will exist between the equilibria and the structure can be reconfigured by transitioning between the two unstable states $E_{1}$ and $E_{2}$.

\subsection{Uncontrolled heteroclinic connection}

The search for heteroclinic connections discussed above is aided by the presence of symmetry in the problem. Indeed, symmetry is often an essential requirement for the existence of heteroclinic connections in dynamical systems. We will therefore impose symmetry on the problem by setting $\mu_{3}=\mu_{1}$. We then note from equations (22) and (24), and indeed from figure 6, that the system then admits the symmetries $\gamma_{1}$ and $\gamma_{2}$ defined by

$$
\gamma_{1}:\left(\begin{array}{c}
q_{1} \\
q_{2}
\end{array}\right) \rightarrow\left(\begin{array}{l}
q_{2} \\
q_{1}
\end{array}\right) \quad \gamma_{2}:\left(\begin{array}{l}
q_{1} \\
q_{2}
\end{array}\right) \rightarrow\left(\begin{array}{l}
-q_{2} \\
-q_{1}
\end{array}\right)
$$

which amount to reflections through $q_{2}=q_{1}$ and $q_{2}=-q_{1}$ respectively. Since $\gamma_{1}$ and $\gamma_{2}$ form a group $\Gamma$, the problem forms a so-called equivariant dynamical system [13]. Then, given that the dynamics of the problem are defined by $\dot{\mathbf{p}}=-\nabla_{q} V(\mathbf{q}, \mu)$, it can be shown that $\nabla_{q} V(\gamma \cdot \mathbf{q}, \mu)=\gamma \cdot \nabla_{q} V(\mathbf{q}, \mu)$ for $\gamma \in \Gamma$. This property of the system ensures that once a solution $\mathbf{q}(t)$ is found, symmetric solutions can also be found using $\gamma_{1}$ and $\gamma_{2}$. Therefore, any solution generated from $\mathbf{q}(t)=\{\gamma \cdot \mathbf{q}(t) \mid \gamma \in \Gamma\}$ is also a valid solution of $\dot{\mathbf{p}}=-\nabla_{q} V(\mathbf{q}, \mu)$. 
To take advantage of these symmetries the coordinate axes $\left(q_{1}, q_{2}\right)$ will be rotated anticlockwise by $\pi / 4$ with the following coordinate transformation

$$
\left(\begin{array}{l}
q_{1} \\
q_{2}
\end{array}\right)=\frac{1}{\sqrt{2}}\left(\begin{array}{cc}
1 & -1 \\
1 & 1
\end{array}\right)\left(\begin{array}{l}
Q_{1} \\
Q_{2}
\end{array}\right)
$$

Then, the potential defined in equation (20) can be transformed to

$$
\begin{aligned}
V(\mathbf{Q}, \mu)= & \frac{1}{8}\left(Q_{1}^{4}+Q_{1}^{2}\left(6 Q_{2}^{2}-2\left(\mu_{1}+\mu_{3}\right)\right)+4 Q_{1} Q_{2}\left(\mu_{1}-\mu_{3}\right)\right. \\
& \left.+Q_{2}^{2}\left(9 Q_{2}^{2}-2\left(\mu_{1}+4 \mu_{2}+\mu_{3}\right)\right)\right)
\end{aligned}
$$

In this new coordinate system, the equations of motion can be obtained from $\dot{\mathbf{P}}=$ $-\nabla_{Q} V(\mathbf{Q}, \mu)$. The dynamics are then described by

$$
\begin{aligned}
& \dot{Q}_{1}=P_{1} \\
& \dot{P}_{1}=-\frac{Q_{1}}{2}\left(Q_{1}^{2}+3 Q_{2}^{2}-2 \mu_{1}\right), \\
& \dot{Q}_{2}=P_{2} \\
& \dot{P}_{2}=-\frac{Q_{2}}{2}\left(3\left(Q_{1}^{2}+3 Q_{2}^{2}\right)-2 \mu_{1}-4 \mu_{2}\right)
\end{aligned}
$$

In this form the equilibria can again be located (with $\mu_{1,2}>0$ ). It can be shown that the origin $E_{0}$ is a double saddle with eigenvalues $\pm \sqrt{\mu_{1}}, \pm \sqrt{\mu_{1}+2 \mu_{2}}$ while $E_{1}$ and $E_{2}$ are located at $\left( \pm \sqrt{2 \mu_{1}}, 0\right)$ and have eigenvalues $\pm i \sqrt{2 \mu_{1}}, \pm \sqrt{2\left(\mu_{2}-\mu_{1}\right)}$. Finally, $E_{3}$ and $E_{4}$ are located at $\left(0, \pm \frac{1}{3} \sqrt{2\left(\mu_{1}+2 \mu_{2}\right)}\right)$ with eigenvalues $\pm i \sqrt{2\left(\mu_{1}+2 \mu_{2}\right)}, \pm \sqrt{\frac{2}{3}\left(\mu_{1}-\mu_{2}\right)}$. The eigenvalues are again obtained by linearisation of equations (32) and (34) at the appropriate equilibrium point.

Using the transformed coordinates, it can be seen that $E_{3}$ and $E_{4}$ will merge with $E_{0}$ for $\mu_{2}<-\frac{1}{2} \mu_{1}$, as shown in figure 8. Similarly, it is clear that the sign of $\mu_{1}-\mu_{2}$ determines the stability of the equilibria, with a bifurcation taking place as $\mu_{1}-\mu_{2}$ crosses zero. Here a pair of conjugate imaginary eigenvalues become real, leading to a transition for a centre to a saddle. Therefore, when $\mu_{1}<\mu_{2}$, the equilibria $E_{1,2}$ are unstable (saddle $\times$ centre) and $E_{3,4}$ are stable (centre $\times$ centre). When $\mu_{1}>\mu_{2}$ these stability properties are reversed, again shown in figure 8 . When $\mu_{1}=\mu_{2}$, the system admits a 'ring equilibrium', as noted earlier. For the numerical investigations that follow, we will let $\mu_{2}>\mu_{1}$, as in this case $E_{1,2}$ are unstable and their position is independent of $\mu_{2}$.

In the rotated coordinates, the system is symmetric about the axes $Q_{1}=0$ and $Q_{2}=0$. The unstable manifold of $E_{1}$, for example, is then the reflection of the stable manifold of $E_{2}$. Therefore, a heteroclinic connection between $E_{1}$ and $E_{2}$, if one exists, must be symmetric about the $Q_{2}$ axis, and so must intersect $Q_{1}=0$ perpendicularly, i.e. $\dot{Q}_{2}=0$, as shown in figures 9 and 10. The numerical method used to find heteroclinic connections is therefore direct: for a certain parameter set, we integrate the system of 

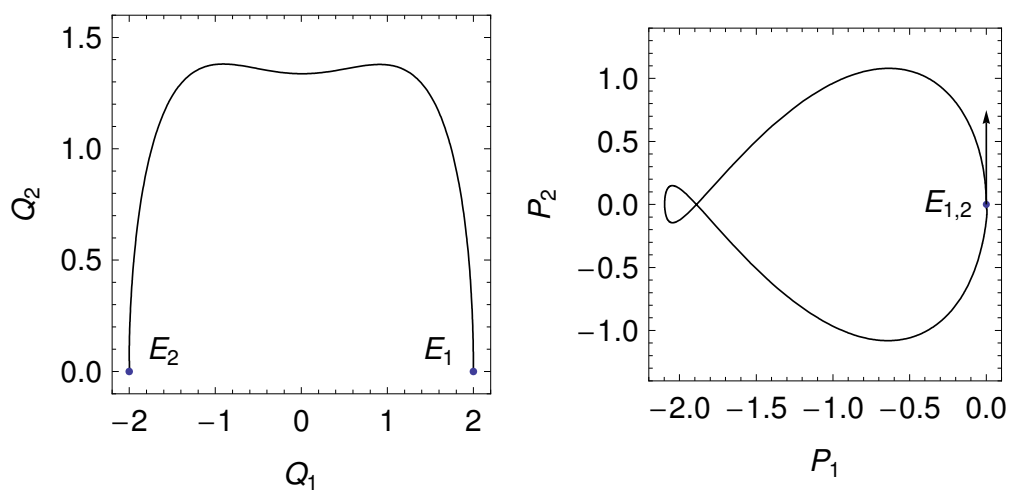

Figure 9. A heteroclinic connection between $E_{1}$ at $(2,0)$ and $E_{2}$ at $(-2,0)$ for $\mu_{1}=2, \mu_{2}=1.68 \mu_{1}$ and $\mu_{3}=2$. The projection of the phase space path into configuration space $\mathbf{Q}$ and momentum space $\mathbf{P}$ is shown. Note the perpendicular crossing of $Q_{1}=0$ with $\dot{Q}_{2}=0$.

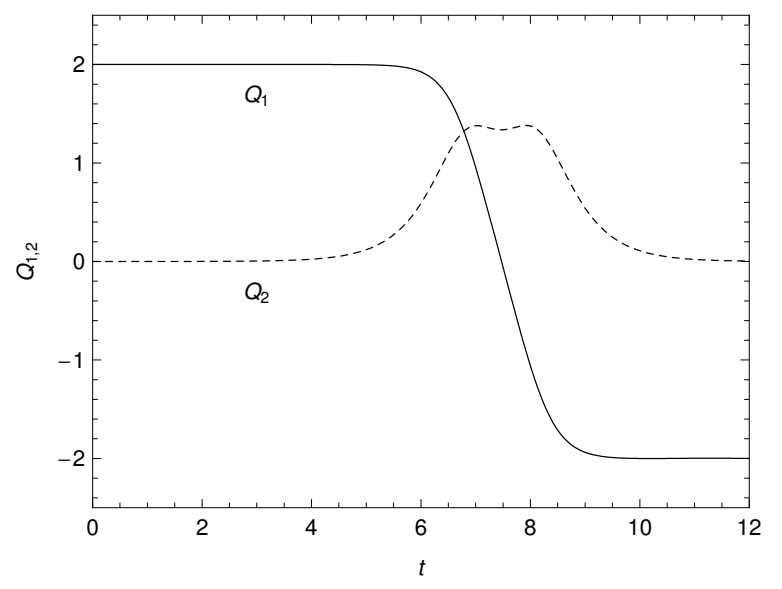

Figure 10. Transformed coordinates $Q_{1}$ and $Q_{2}$ for a heteroclinic connection between $E_{1}$ at $(2,0)$ and $E_{2}$ at $(-2,0)$ for $\mu_{1}=2, \mu_{2}=1.68 \mu_{1}$ and $\mu_{3}=2$.

equations in the direction of the unstable eigenvector of $E_{1}$ as in equation (27), until it intersects the $Q_{2}$-axis, i.e. $Q_{1}=0$. We measure $\dot{Q}_{2}$ at this time, and if $\dot{Q}_{2}=0$ (or less than some cut-off) a heteroclinic connection exists between $E_{1}$ and $E_{2}$ for this parameter set. Due to symmetry, a heteroclinic connection will also exist between $E_{2}$ and $E_{1}$, and both connections will have a mirror image under $Q_{2} \rightarrow-Q_{2}$. This is a consequence of the dynamics of the problem being equivariant.

As noted earlier, a true heteroclinic path is an asymptotic connection. It therefore reaches the equilibrium points as $t \rightarrow \pm \infty$. This is clearly impractical for a real structure. Therefore, we must instead approximate the true heteroclinic paths with approximate heteroclinic paths, by which we mean the path enters the close vicinity of both fixed points in finite time. For this reason, we do not require $\dot{Q}_{2}$ to be exactly zero on crossing $Q_{1}=0$, merely to be smaller than some chosen value. 


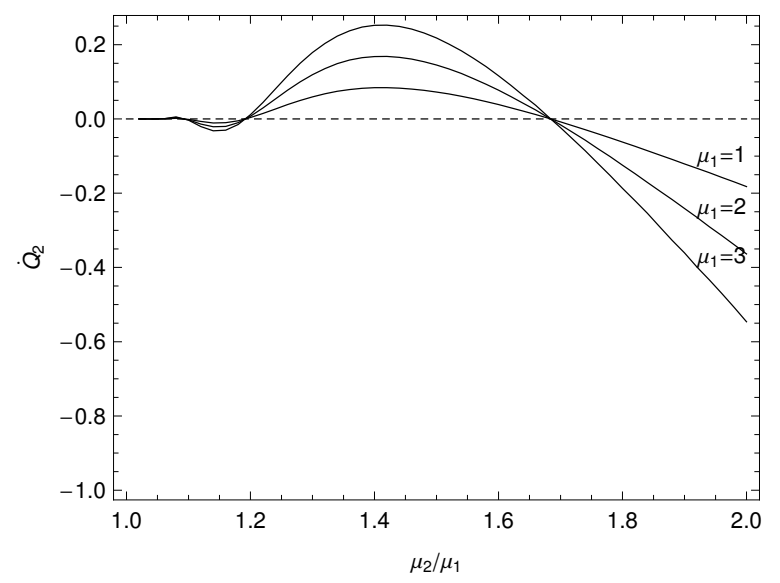

Figure 11. The value of $\dot{Q}_{2}$ at the first crossing of the unstable manifold of $E_{1}$ with the $Q_{2}$ axis, with increasing parameter ratio $\mu_{2} / \mu_{1}$.

From the transformed equations of motion it can again be seen that when $\mu_{1}=\mu_{2}$ there is a continuous ring of equilibria which, from equations (32) and (34), is the ellipse defined by $Q_{1}^{2}+3 Q_{2}^{2}=2 \mu$. For $\mu_{2}>\mu_{1}$ the symmetry of this continuous ring of equilibria is broken into the four equilibria $E_{1-4}$. Once this symmetry is broken the connection between $E_{1}$ and $E_{2}$ can persist in the form of a heteroclinic connection. Numerically, it is found that for $\mu_{2}<1.2 \mu_{1}, \dot{Q}_{2}$ is sufficiently small for an approximate heteroclinic path to exist, as shown in figure 11 . Then, when $\mu_{2} \approx 1.68 \mu_{1}$, a heteroclinic path exists, irrespective of the value of $\mu_{1}$, as is clearly seen in figure 11. This demonstrates that for each value of $\mu_{1}$ there is a value of $\mu_{2}$ not close to $\mu_{1}$ which admits a heteroclinic path. Lastly, instead of measuring the value of $\dot{Q}_{2}$ on the first intersection with $Q_{1}=0$, we could instead continue the integration until subsequent intersections. We find that there are more complicated heteroclinic paths which leave $E_{1}$, intersect the $Q_{2}$ axis a number of times, and then approach $E_{2}$. While these paths are of interest, their more complicated form suggests they are more likely to be destroyed by damping or parameter inaccuracy, and so will not be discussed further here. We may conclude that exact heteroclinic paths do not exist for every choice of parameters $\mu_{1}$ and $\mu_{2}$. However, we can overcome this shortcoming by using a controller, which we shall discuss in the next section.

\subsection{Controlled heteroclinic connection}

It has been shown that the 2 mass chain admits families of heteroclinic connections in the phase space of the problem. These families of trajectories rely on both the symmetry and the Hamiltonian structure of the dynamics. For a more realistic model however, dissipation must be considered, which will of course destroy the Hamiltonian structure of the dynamics. Therefore, phase trajectories emerging from $E_{1}$ will not reach $E_{2}$. To compensate for such dissipation, a controller will now be defined which captures phase trajectories in a neighbourhood $R$ of $E_{2}$. In addition, a controller is clearly required to 
ensure stability at $E_{1}$, prior to initiation of the heteroclinic connection and for continued operation at $E_{2}$ following the transition.

The dynamics of the problem will now be extended by the addition of linear dissipation parameterised by $\beta$. Then, returning to the original variables $\left(q_{1}, q_{2}\right)$ for ease of illustration, the dynamics are defined by

$$
\begin{aligned}
& \dot{q_{1}}=p_{1} \\
& \dot{p_{1}}=\mu_{1} q_{1}-q_{1}^{3}+\mu_{2}\left(q_{1}-q_{2}\right)-\left(q_{1}-q_{2}\right)^{3}-\beta p_{1} \\
& \dot{q_{2}}=p_{2} \\
& \dot{p_{2}}=\mu_{3} q_{2}-q_{2}^{3}-\mu_{2}\left(q_{1}-q_{2}\right)+\left(q_{1}-q_{2}\right)^{3}-\beta p_{2}
\end{aligned}
$$

It can then be shown that in general $\left(p_{1} \neq 0, p_{2} \neq 0\right)$ the total energy $W=T+V$ of the system is monotonically decreasing such that $\dot{W}=-\beta\left(p_{1}^{2}+p_{2}^{2}\right)$. In order to define a suitable controller it will be assumed that the states $\mathbf{q}=\left(q_{1}, q_{2}\right)$ and $\mathbf{p}=\left(p_{1}, p_{2}\right)$ can be observed. Similarly, in order to effect control, it will be assumed that the coupling parameters $\mu_{1-3}$ can be manipulated. This is equivalent to manipulating the natural length of the springs in the model. As will be seen, the system is controllable through the manipulation of $\mu_{1}$ and $\mu_{3}$ only, with $\mu_{2}$ fixed.

In order to ensure convergence to some equilibrium point $\left(\widetilde{q_{1}}, \widetilde{q_{2}}\right)$ a Lyapunov function [24] will be defined as

$$
\phi(\mathbf{q}, \mathbf{p})=\frac{1}{2}\left(p_{1}+p_{2}\right)^{2}+\frac{1}{2}\left(q_{1}-\widetilde{q_{1}}\right)^{2}+\frac{1}{2}\left(q_{2}-\widetilde{q_{2}}\right)^{2}
$$

where $\phi(\mathbf{q}, \mathbf{p})>0$ and $\phi(0,0)=0$. The time derivative of the Lyapunov function is clearly

$$
\dot{\phi}(\mathbf{q}, \mathbf{p})=p_{1}\left(\dot{p_{1}}+\left(q_{1}-\widetilde{q_{1}}\right)\right)+p_{2}\left(\dot{p_{2}}+\left(q_{2}-\widetilde{q_{2}}\right)\right)
$$

Then, substituting from equations (36) and (38) the following controllers for $\mu_{1}$ and $\mu_{3}$ can be defined $\left(q_{1,2} \neq 0\right)$ as

$$
\begin{aligned}
& \mu_{1}=-\frac{1}{q_{1}}\left(\left(q_{1}-\widetilde{q}_{1}\right)+\eta p_{1}+q_{1}-q_{1}^{3}+\mu_{2}\left(q_{1}-q_{2}\right)+\left(q_{1}-q_{2}\right)^{3}\right) \\
& \mu_{3}=-\frac{1}{q_{2}}\left(\left(q_{2}-\widetilde{q}_{2}\right)+\eta p_{2}+q_{2}-q_{2}^{3}-\mu_{2}\left(q_{1}-q_{2}\right)+\left(q_{1}-q_{2}\right)^{3}\right)
\end{aligned}
$$

for some control parameter $\eta$. It can be seen that $\phi$ is monotonically decreasing such that

$$
\dot{\phi}(\mathbf{q}, \mathbf{p})=-(\eta+\beta)\left(p_{1}^{2}+p_{2}^{2}\right) \preceq 0
$$

and so $\mathbf{q} \longrightarrow\left(\widetilde{q_{1}}, \widetilde{q_{2}}\right)$ and $\mathbf{p} \longrightarrow(0,0)$ within the neighbourhood $R$ of $E_{2}$. If the neighbourhood $R$ of $E_{2}$ is sufficiently small, it can also be shown that the controls can be approximated to simple linear feedback of the form 


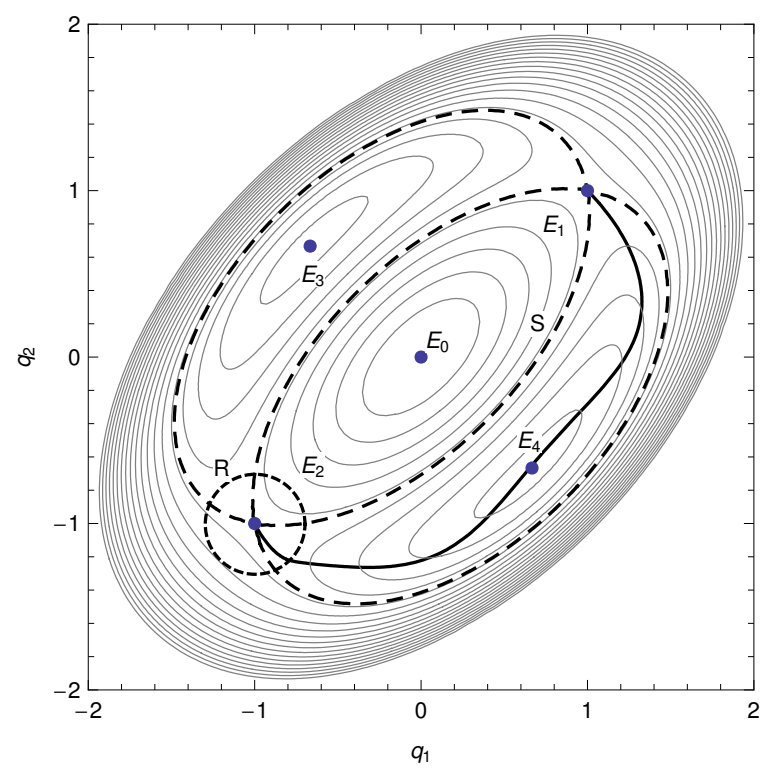

Figure 12. Controlled transition from $E_{1}$ at $(1,1)$ to $E_{2}$ at $(-1,-1)$ with the controller active in the neighbourhood $R$ of $E_{2}$ (dissipation parameter $\beta=0.01$ ). Contour $S$ represents the allowed region of motion with $T(\mathbf{p})>0$.

$$
\begin{aligned}
\delta \mu_{1} & =\frac{1}{2} \delta q_{1}-\frac{3}{2} \delta q_{2}+\eta p_{1} \\
\delta \mu_{3} & =-\frac{3}{2} \delta q_{1}+\frac{1}{2} \delta q_{2}+\eta p_{2}
\end{aligned}
$$

where $\left(\delta q_{1}, \delta q_{2}\right)=\left(q_{1}-\widetilde{q}_{1}, q_{2}-\widetilde{q}_{2}\right)$. We note from equation (43) that $\dot{\phi}(\mathbf{q}, \mathbf{p})$ is only rendered negative semi-definite and so asymptotic stability within $R$ is not ensured from equation (43) alone. However, the Krasovskii-LaSalle principle [24] can be invoked to demonstrate asymptotic stability. It can be seen that both $\phi(0,0)=0$ and $\dot{\phi}(0,0)=0$. In addition the set $\{(\mathbf{q}, \mathbf{p}) \mid \dot{\phi}(\mathbf{q}, \mathbf{p})=0\}$ does not contain any trajectory of the system, except the trivial trajectory $\mathbf{q}=\mathbf{p}=0$ within $R$, so that asymptotic stability is ensured.

An example of a controlled heteroclinic connection for $\mu_{1}=1, \mu_{2}=1.5$ and $\mu_{3}=1$ is shown in figure $12\left(\beta=10^{-2}\right)$. In the absence of dissipation, the Hamiltonian $H=T(\mathbf{p})+V(\mathbf{q}, \mu)$ is constant so that the volume of phase space in $\mathbf{R}^{4}$, and its projection to configuration space in $\mathbf{R}^{2}$, is constrained by the requirement that $T(\mathbf{p}) \geq 0$, identified by contour $S$ in figure 12. Since the ratio of $\mu_{1}$ and $\mu_{2}$ is not close to 1.68 , an exact heteroclinic connection will not occur (in the absence of dissipation), as can be seen from figure 11. To initiate the heteroclinic connection, a displacement along the unstable manifold of $E_{1}$ is performed $\left(\epsilon=10^{-4}\right)$ and the controller activated when the phase path is in the neighbourhood $R$ of $E_{2}(\eta=2)$. The corresponding shape of the structure during the transition from $E_{1}$ to $E_{2}$ is shown in figure 13.

The heteroclinic connection can also be seen in figure 14, where the controller ensures capture and stabilisation at $E_{2}$. The corresponding controls $\mu_{1}$ and $\mu_{3}$ are 

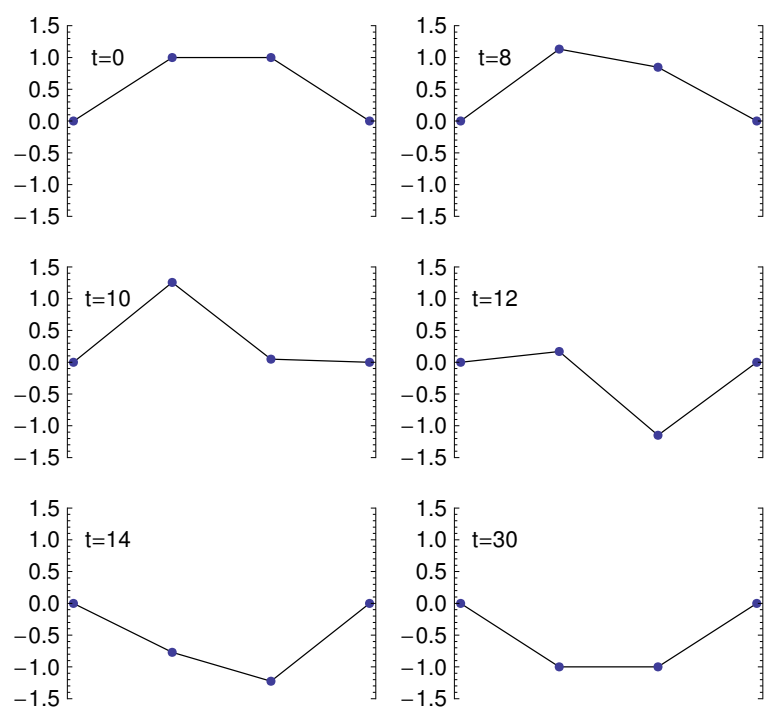

Figure 13. Controlled transition from unstable equilibrium $E_{1}$ at $t=0$ to unstable equilibrium $E_{2}$ at $t=30$.

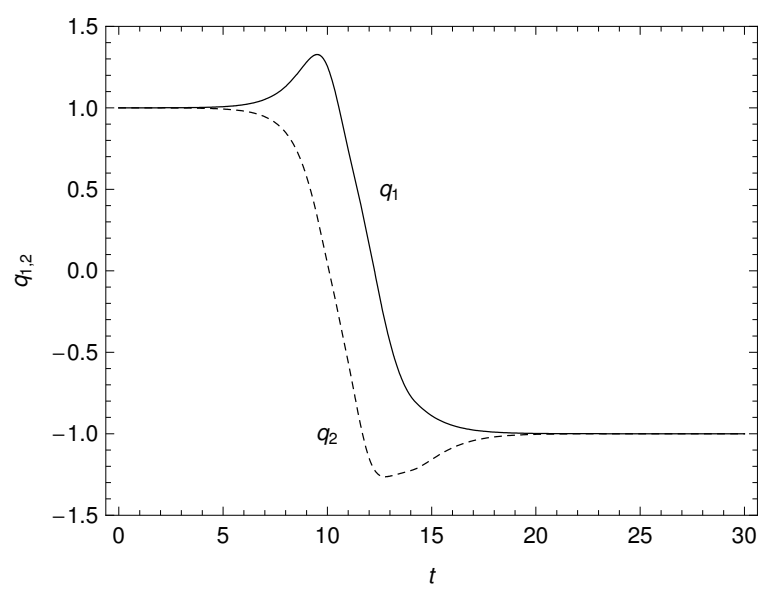

Figure 14. Mass displacements during the transition form $E_{1}$ at $(1,1)$ to $E_{2}$ at $(-1,-1)$.

shown in figure 15. It can be seen that the controls are only active when the phase path is within region $R$ of $E_{2}$ and that a smooth control time history is achieved. Numerical experiments demonstrate that the integrated control effort grows with increasing dissipation parameter $\beta$. Indeed, increasing $\beta$, the region $R$ needs to be enlarged to ensure capture of the phase path at $E_{2}$, as shown in figure 16 . In addition, the duration of the reconfiguration manoeuvre can be reduced by providing a larger displacement $\epsilon$ along with unstable eigenvector of $E_{1}$ defined in equation (27), as shown in figure 17. 


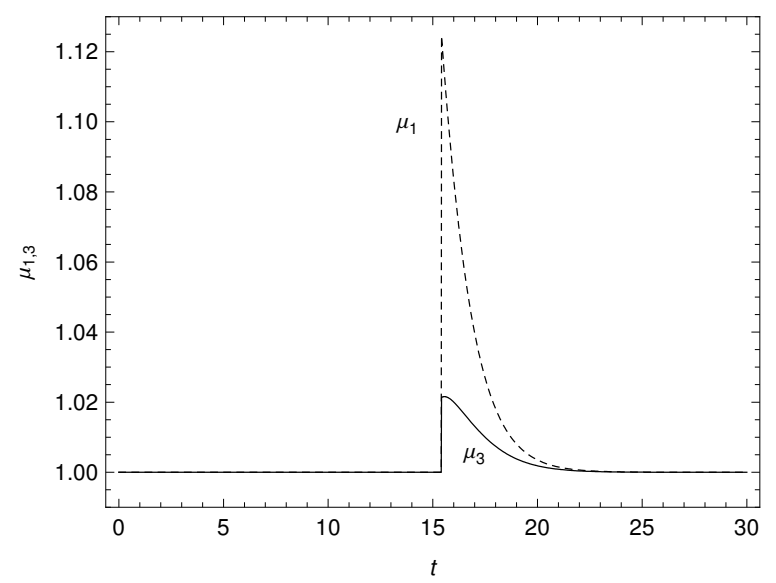

Figure 15. Controls in region $R$ in the neighbourhood of $E_{2}$ actuated through the coupling parameters $\mu_{1}$ and $\mu_{3}$ with $\eta=2$.

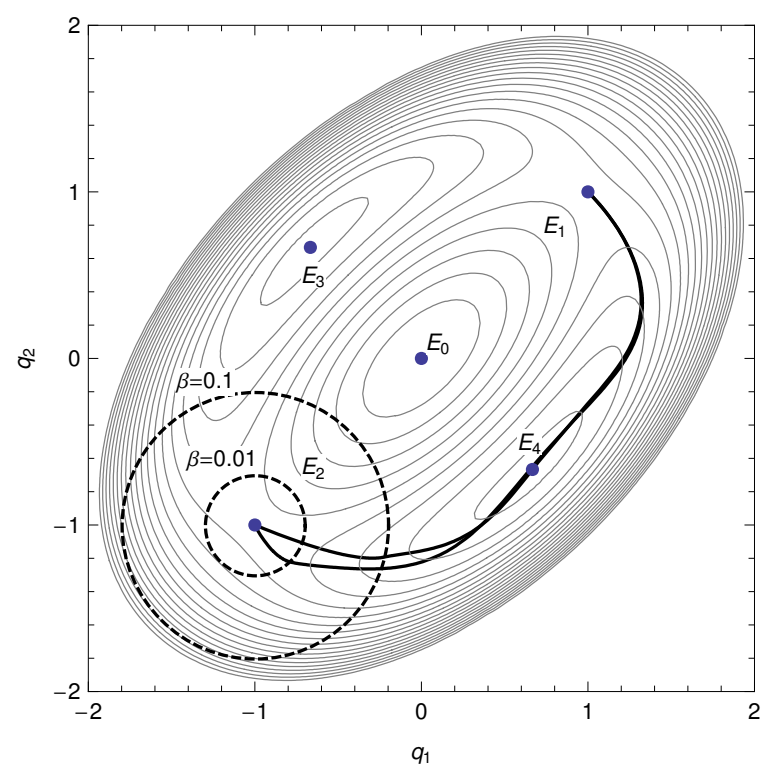

Figure 16. Controlled transition from $E_{1}$ at $(1,1)$ to $E_{2}$ at $(-1,-1)$ with the controller active in the neighbourhood $R$ of $E_{2}$ (dissipation parameter $\beta=0.01, \beta=0.1$ ).

\subsection{Bifurcation control}

As note earlier, although the use of heteroclinic connections appears attractive, work is required to stabilise the structure when operating in the unstable configurations $E_{1}$ and $E_{2}$. However, this limitation may be overcome if the free parameters $\mu_{1-3}$ are selected such that the structure is operating near the critical region of the bifurcation diagram in figure 8 when $\mu_{2} \sim 1$. For $\mu_{2}<1$ the equilibria $E_{1}$ and $E_{2}$ are stable, and the potential forms local minima at these locations, as shown in figure 18. We note that the location of $E_{1}$ and $E_{2}$ are independent of $\mu_{2}$. Then, if $\mu_{2}$ is increased such that $\mu_{2}>1$, the equilibria $E_{1}$ and $E_{2}$ become unstable and a heteroclinic connection 


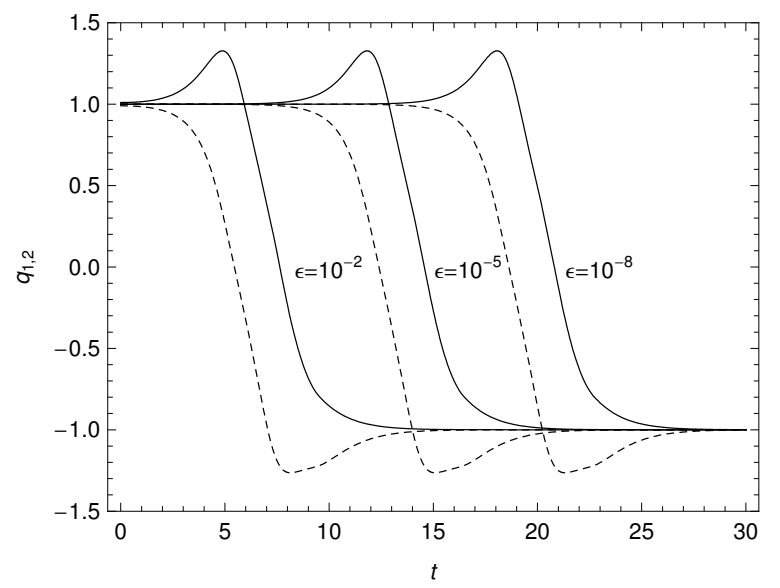

Figure 17. Mass displacements during the transition form $E_{1}$ at $(1,1)$ to $E_{2}$ at $(-1,-1)$ for $\epsilon=10^{-2}, 10^{-5}, 10^{-8}$.

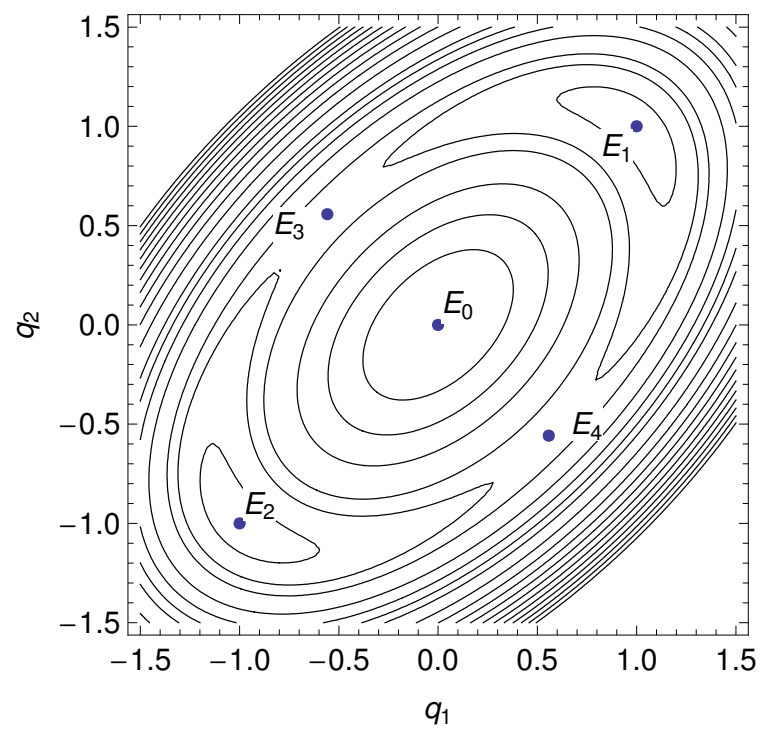

Figure 18. Effective potential $V(\mathbf{q}, \mu)$ for a two mass chain with $\mu_{1}=1, \mu_{2}=0.9$ and $\mu_{3}=1 . E_{1}$ and $E_{2}$ are stable, $E_{3}$ and $E_{4}$ are unstable.

can be used to reconfigure the structure, as shown in figure 19. After the transition from $E_{1}$ to $E_{2}, \mu_{2}$ is finally decreased such that $\mu_{2}<1$ and the equilibria $E_{1}$ and $E_{2}$ again stable. This scheme then allows normal operation in a stable mode, with a transition to instability for reconfiguration of the structure and then a return to stability for continued operation. A transition using this scheme (with no dissipation) is shown in figure 20. The coupling parameters for the end springs are fixed with $\mu_{1}=1$ and $\mu_{3}=1$. The initial oscillation of the system in the potential well at $E_{1}$ with $\mu_{2}<1$ can be seen, followed by a transition to $E_{2}$ with $\mu_{2}>1$ and then a return to oscillation in the potential well at $E_{2}$ with $\mu_{2}<1$. With this scheme, the heteroclinic connection is used for the transition, but the normal operating mode of the structure is stable. 


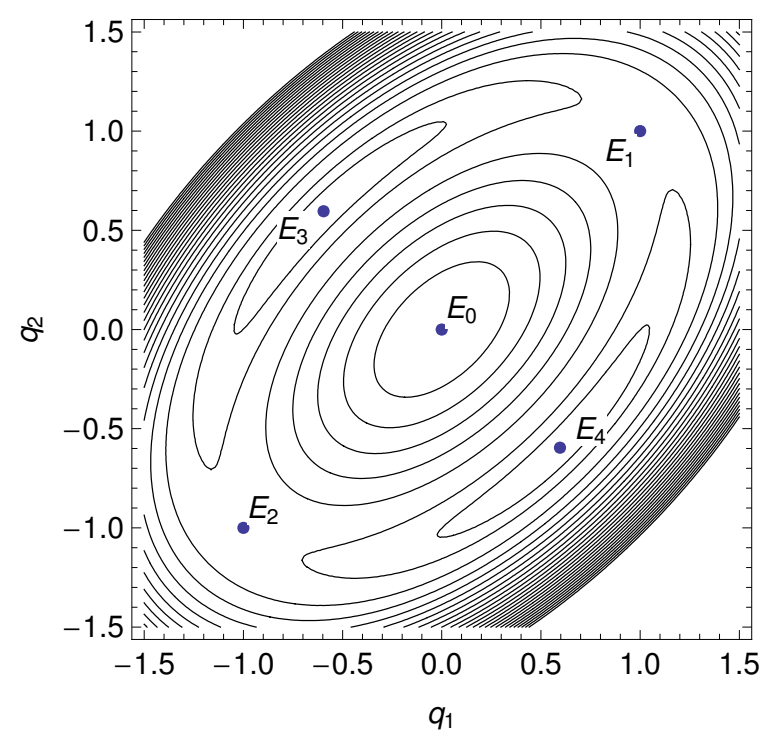

Figure 19. Effective potential $V(\mathbf{q}, \mu)$ for a two mass chain with $\mu_{1}=1, \mu_{2}=1.1$ and $\mu_{3}=1 . E_{1}$ and $E_{2}$ are unstable, $E_{3}$ and $E_{4}$ are stable.

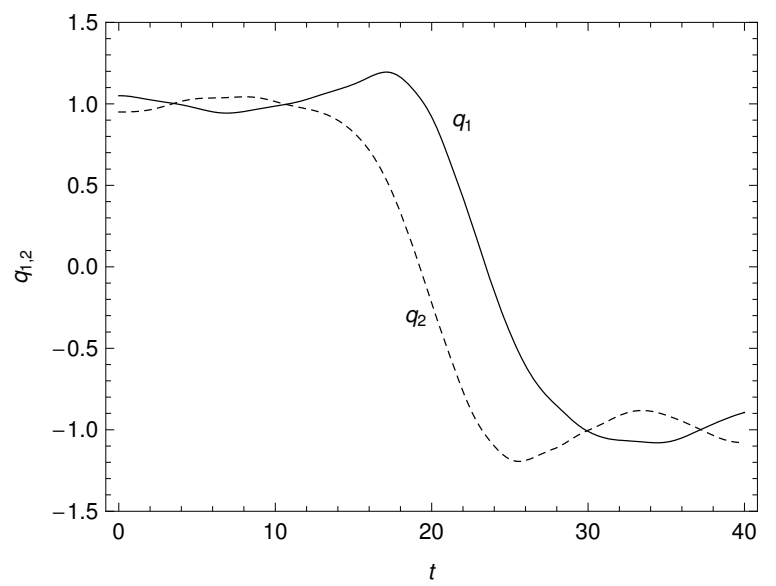

Figure 20. Controlled transition from $E_{1}$ at $(1,1)$ to $E_{2}$ at $(-1,-1)$ with bifurcation control. The coupling parameters $\mu_{1}=1$ and $\mu_{3}=1$ with $\mu_{2}$ switched from 1.1 to 0.9 to manipulate the stability properties of $E_{1}$ and $E_{2}$.

\section{Conclusions}

A new concept for the active reconfiguration of smart structures has been presented using the powerful concept of phase space connections. Using a simple, representative model of an unstable structure it has been demonstrated that the unstable (but controllable) equilibrium configurations of such a structure can be connected. In principle, such reconfigurations do not require the input of energy, other than to overcome dissipation in the system. This is in contrast to conventional structures which require energy input and dissipation to transition between passively stable configurations. While the model used is simplistic, it provides insights into the problem which can be exploited to develop 
the concept towards the reconfiguration of real smart structures.

\section{Acknowledgments}

Acknowledgments This work has been partly supported by the Engineering and Physical Science Research Council grant EP-D003822-1.

\section{References}

[1] I. Chopra. Review of state of art of smart structures and integrated systems. AIAA Journal, 40(11):2145-2187, 2002.

[2] J. Welham, E. P. Calius, and J. G. Chase. Active stabilization of thin-wall structures under compressive loading. In A. M. Baz, editor, Smart Structures and Materials 2003: Smart Structures and Integrated Systems. Edited by Baz, Amr M. Proceedings of the SPIE, Volume 5056, pp. 229-240 (2003)., volume 5056 of Presented at the Society of Photo-Optical Instrumentation Engineers (SPIE) Conference, pages 229-240, August 2003.

[3] C. Hwu, W. C. Chang, and H. S. Gai. Vibration suppression of composite sandwich beams. Journal of Sound Vibration, 272:1-2, April 2004.

[4] T. Meressi and B. Paden. Buckling control of a flexible beam using piezoelectric actuators. Journal of Guidance Control Dynamics, 16:977-980, October 1993.

[5] G. Venkateswara Rao and G. Singh. TECHNICAL NOTE: A smart structures concept for the buckling load enhancement of columns. Smart Material Structures, 10:843-845, August 2001.

[6] T. Hogg and B.A. Huberman. Controlling smart matter. Journal of Smart Materials and Structures, 7(1):R1-R14, 1998.

[7] O. Guenther, T. Hogg, and B. A. Huberman. Controls for unstable structures. In V. V. Varadan and J. Chandra, editors, Proc. SPIE Vol. 3039, p. 754-763, Smart Structures and Materials 1997: Mathematics and Control in Smart Structures, Vasundara V. Varadan; Jagdish Chandra; Eds., volume 3039 of Presented at the Society of Photo-Optical Instrumentation Engineers (SPIE) Conference, pages 754-763, June 1997.

[8] O. Guenther, T. Hogg, and B. A. Huberman. Pattern control in unstable structures. In V. V. Varadan, editor, Proc. SPIE Vol. 3323, p. 436-445, Smart Structures and Materials 1998: Mathematics and Control in Smart Structures, Vasundara V. Varadan; Ed., volume 3323 of Presented at the Society of Photo-Optical Instrumentation Engineers (SPIE) Conference, pages 436-445, July 1998.

[9] D. W. Jordan and P. Smith. Nonlinear Ordinary Differential Equations: An Introduction to Dynamical Systems. Oxford University Press, Oxford, 1999.

[10] V. G. A. Goss, G. H. M. van der Heijden, J. M. T. Thompson, and S. Neukirch. Experiments on snap buckling, hysteresis and loop formation in twisted rods. Experimental mechanics, 10(2):101-111, 2000.

[11] W. S. Koon, M. W. Lo, J. E. Marsden, and S. D. Ross. Heteroclinic connections between periodic orbits and resonance transitions in celestial mechanics. Chaos, 45(2):427-469, 2005.

[12] D. Taylor and P. Holmes. Simple models for excitable and oscillatory neural networks. Journal of Mathematical Biology, 37(5):419-446, 1998.

[13] M. Aguiar, S. Castro, and I. Labouriau. Dynamics near a heteroclinic network. Nonlinearity, 18(1):391-414, 2005.

[14] H. Su and J. M. McCarthy. A polynomial homotopy formulation for the inverse static analysis of planar compliant mechanisms. Transactions of the ASME, 128(4):776-786, 2006.

[15] S.D. Guest and S. Pellegrino. Analytical models for bistable cylindrical shells. Proceedings of the Royal Society A, 462(3):839-854, 2006. 
[16] S. Liu, A. Davidson, and Q. Lin. Simulation studies on nonlinear dynamics and chaos in a mems cantilever control system. Journal of Micromechanics and Microengineering, 14:1064-1073, 2004.

[17] J. Guo and Y. Zhao. Dynamic stability of electrostatic torsional actuators with van der waals effect. International Journal of Solids and Structures, 43(3-4):675-685, 2006.

[18] J. Qiu, J.H. Land, and A. H. Solcum. A curved-beam bistable mechanism. Journal of microelectromechanical systems, 13(2):137-146, 2004.

[19] R. C. Merkle. Two types of mechanical reversible logic. Nanotechnology, 4(2):114-131, April 1993.

[20] J. Loughlan, S.P. Thompson, and H. Smith. Buckling control using embedded shape memory actuators and the utilisation of smart technology in future aerospace platforms. Composite Structures, 58(3):319-347, 2002.

[21] M. Carpel and B. Moulin. Models for aeroservoelastic analysis with smart structures. Journal of Aircraft, 41(2):314-321, 2004.

[22] S. H. Strogatz. Nonlinear Dynamics and Chaos. Perseus Books Publishing, Cambridge MA, 1994.

[23] I. V. Andrianova and J. Awrejcewicz. Continuous models for chain of inertially linked masses. European Journal of Mechanics - A/Solids, 24(3):532-536, 2005.

[24] S. Wiggins. Introduction to Applied Nonlinear Dynamical Systems and Chaos. Springer-Verlag, New York, 1997. 